\title{
Toponimi e identità: la percezione dei parlanti valdostani
}

Parte seconda

Luisa Revelli

\section{OpenEdition \\ Journals}

Edizione digitale

URL: http://journals.openedition.org/esp/95

DOI: $10.4000 /$ esp.95

ISSN: 2532-0319

\section{Editore}

Centre d'Information sur l'Éducation Bilingue et Plurilingue

Edizione cartacea

Data di pubblicazione: 1 giugno 2014

Paginazione: 15-23

ISSN: 1127-266X

Notizia bibliografica digitale

Luisa Revelli, « Toponimi e identità: la percezione dei parlanti valdostani », Éducation et sociétés plurilingues [Online], 36 | 2014, Messo online il 25 octobre 2014, consultato il 30 avril 2019. URL : http:// journals.openedition.org/esp/95; DOI : 10.4000/esp.95 


\section{TOPONIMI E IDENTITÀ: LA PERCEZIONE DEI PARLANTI VALDOSTANI. (PARTE SECONDA)}

\section{Luisa REVELLI}

À partir des données d'une enquête menée dans la Vallée d'Aoste afin d'étudier les différentes réalisations phonétiques de certains noms de lieux locaux, la première partie de notre contribution (publiée pp. 15-28 d'ESP $n^{\circ}$ 35, décembre 2013) s'est proposée de résumer les attitudes des locuteurs envers la variété de prononciations, en discutant les opinions, évaluations et sentiments activés par la présence de solutions alternatives. La deuxième partie, publiée ici, conclut la présentation des principaux résultats de l'enquête en approfondissant le rôle joué par l'interprétation des représentations polymorphes, représentations qui ne semblent pas influer sur le comportement linguistique réel, mais qui agissent certainement sur la perception du côté positif ou négatif de l'abondance des variantes concurrentes - alternativement décrites comme spontanées et naturelles, comme événement désagréable ou comme ressource à préserver - et qui, en parallèle, semblent interprétables en termes d'images du plurilinguisme.

Taking off from a survey carried out in the Aosta Valley to study the different phonetic actualizations of some local place-names, the first part of our contribution (published pp. 15-28, ESP $n^{\circ} 35$, December 2013) summed up the attitudes of speakers towards the variety of pronunciations, by discussing the opinions, evaluations and feelings prompted by the presence of alternative solutions. The second part, published here, concludes our presentation of the main results of the survey by an in-depth analysis of the role played by the interpretation of polymorphic representations, which does not seem to influence people's actual linguistic behavior, but which certainly colors their perception of the positive or negative value of the quantity of varieties in competition - alternately described as spontaneous and natural, as disagreeable occurrences or as a resource to be preserved and protected - and which at the same time can be interpreted in terms of images of plurilingualism.

\section{DIALETTOFONIA E REATTIVITÀ}

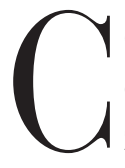

ome anticipato nella prima parte del contributo, nel corso delle interviste è in più occasioni emersa la tendenza degli intervistati a interpretare vere o presunte buone pronunce come manifestazioni di lealtà culturale al territorio. In alcuni casi tale tendenza è stata espressa attraverso asserti del tipo per me (la pronuncia italianizzante) è una cosa negativa perché io sono legata al territorio [P23] o io ci tengo ad essere valdostana e mi piace che... che [i nomi dei paesi] vengano pronunciati nel modo giusto ([P98]). In altri casi la presenza di un legame biunivoco e simbolico tra pronunce, senso di appartenenza e legame al territorio è rimasta sottintesa. Con l'obiettivo di farla emergere quando implicita o 
Toponimi e identità: la percezione dei parlanti valdostani.

L. REVELLI sommersa, sono stati inseriti nel questionario due quesiti che chiedevano di stimare rispettivamente il proprio grado di conoscenza del territorio regionale e il livello di affezione allo stesso. In entrambi i casi per l'autovalutazione era prevista l'attribuzione di un voto compreso tra 1 (per niente) e 4 (molto).

Va notato preliminarmente che le risposte ai due quesiti mostrano un andamento parallelo: le valutazioni sono elevate in entrambi i casi, ma il legame al territorio riceve quasi da tutti un punto in più rispetto alla conoscenza dello stesso; il 65\% del campione considera buona (3 p.) la propria conoscenza della Valle d'Aosta e dichiara di sentirsi ad essa molto legato (4 p.). L'età sembra incidere, seppur moderatamente, sul tenore delle risposte: sono in generale gli anziani ad attribuirsi le valutazioni migliori.

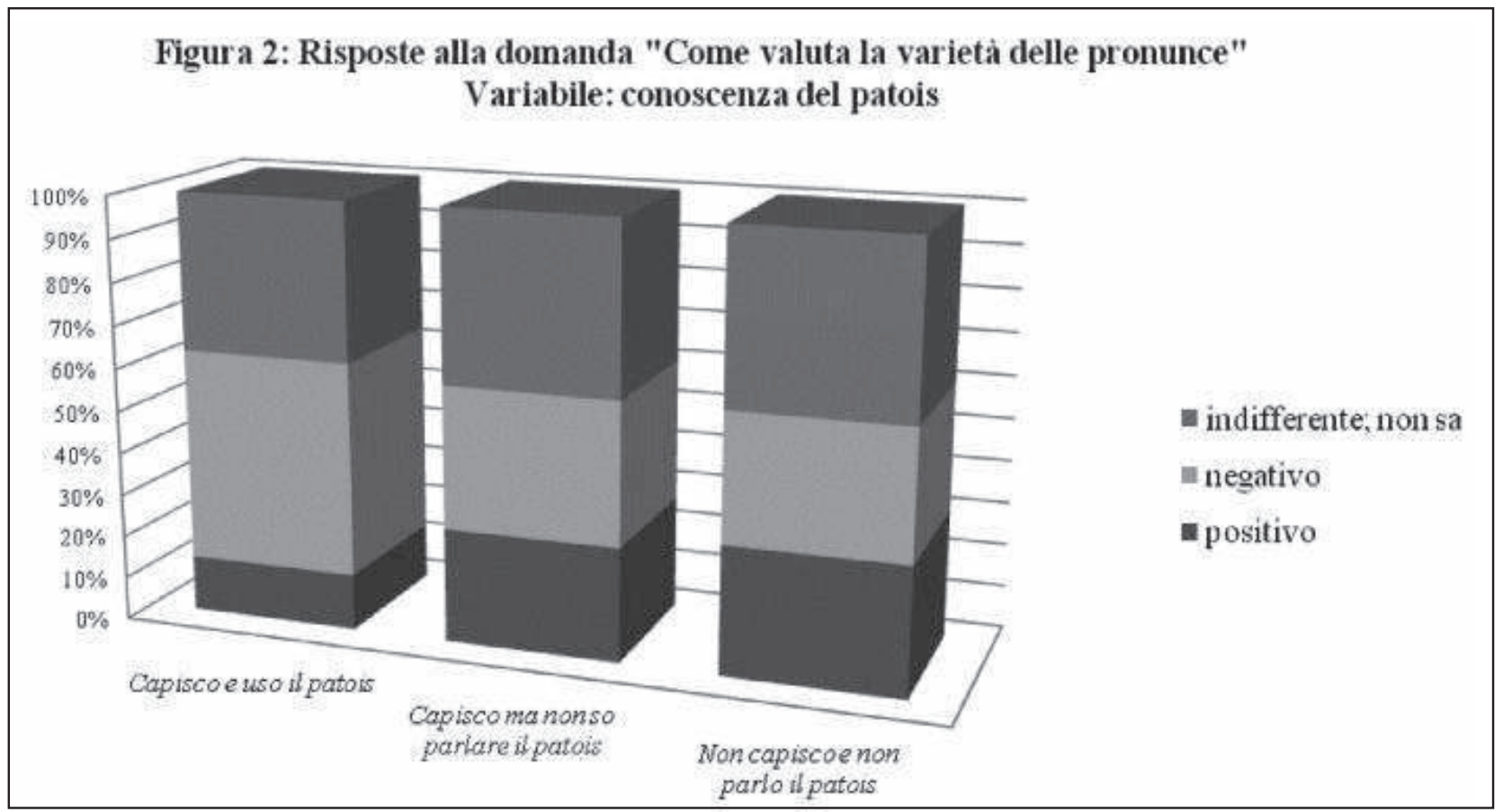

Come prevedibile, un'elevata autovalutazione del proprio legame al territorio appare strettamente correlata anche alla conoscenza del patois: coloro che dichiarano di capire e usare la parlata locale esprimono un valore medio di attaccamento geografico pari a 3,84 (capisco ma non uso il patois: 3,5; non capisco e non uso il patois: 3,13).

L'utilizzo effettivo del patois trova, d'altra parte, corrispondenza in una valutazione prevalentemente negativa della polimorfia delle pronunce: 


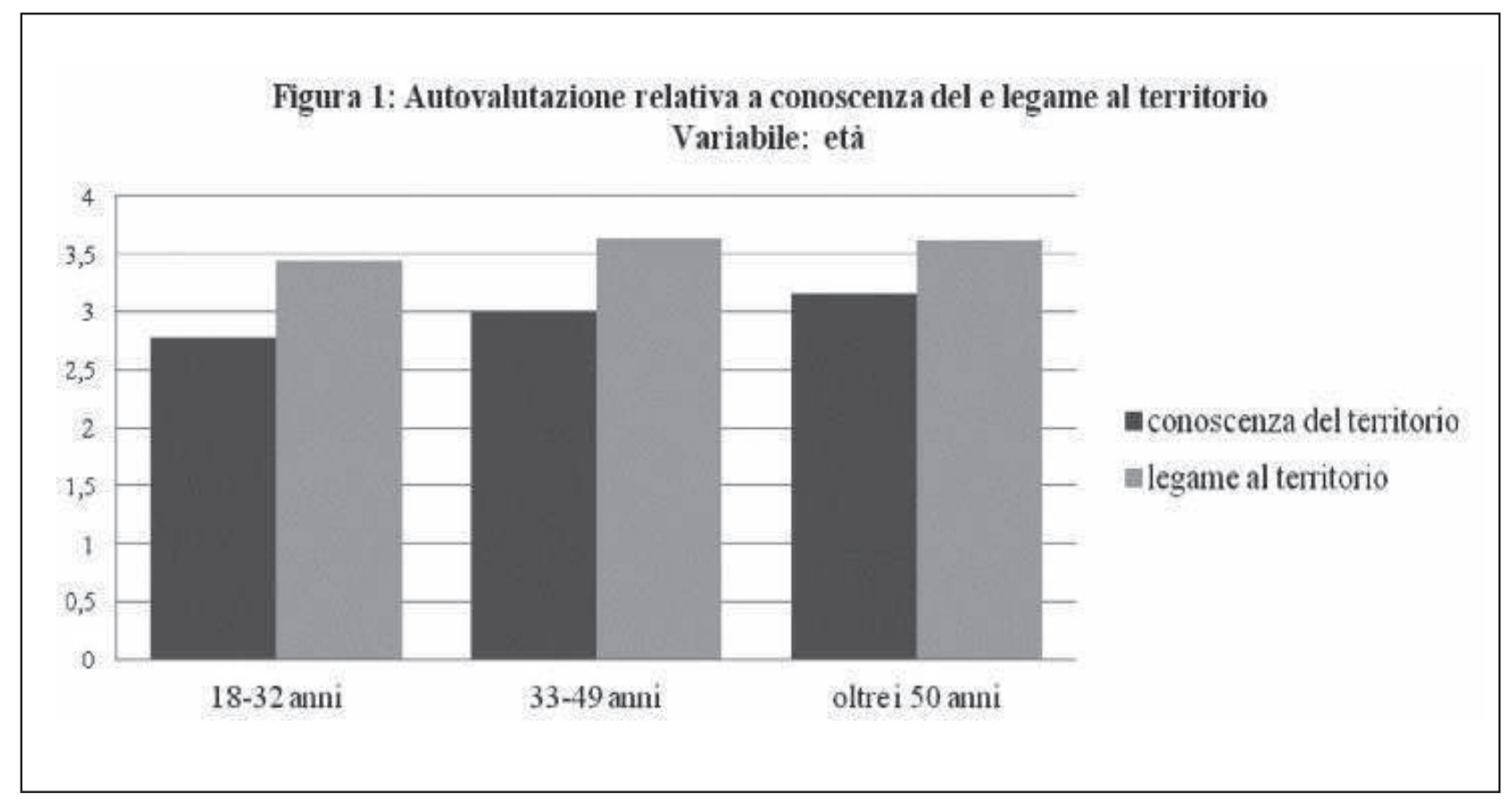

Toponimi e identità: la percezione dei parlanti valdostani.

L. REVELLI

SENSO DELLA DIACRONIA E CAUSE DELLA POLIMORFIA
I giudizi espressi in relazione alla varietà non possono tuttavia condurre a conclusioni univoche: come vedremo nelle pagine seguenti, gli atteggiamenti di maggiore o minore tolleranza si differenziano, infatti, in modo significativo a seconda che le ragioni della polimorfia vengano ricondotte a motivazioni - storiche e di matrice endogena - di diatopia interna o invece interpretate come più o meno recente risultato di fenomeni di contatto con l'italiano e con altre varietà dialettali.

Con un quesito, posto volutamente in modo generico ("Secondo lei come mai uno stesso nome di Comune può essere pronunciato in modi diversi?"), ci si è proposti di rilevare le congetture dei parlanti a proposito delle ragioni della polimorfia toponomastica, al contempo sollecitando l'emergere di suggestioni o condizionamenti almeno in parte rivelatori delle rappresentazioni più intrinsecamente associate ai concetti di plurilinguismo, varietà linguistica, identità e alterità culturale.

Assumendo una prospettiva storica, un numero ridotto di parlanti ha descritto il fenomeno come connaturato alla tradizione plurilingue dell'area e alle politiche linguistiche avvicendatesi nel corso del tempo:

[P125] la Valle, si sa..da sempre... storicamente è sempre stata attraversata da tutti $i$ popoli possibili e immaginabili... quindi pensare di riuscire ad avere... avendo poi il bilinguismo, avendo il patois che ha influenzato tanto... 
Toponimi e identità: la percezione dei parlanti valdostani.

L. REVELLI
[P 102] questa variazione delle pronunce testimonia di un modo di parlare che risente di ... di epoche storiche. Per tanto tempo, penso fino agli anni Ottanta, si tendeva a pronunciarli all'italiana, poi l'intervento della Regione, il cambio della segnaletica da parte dell'Anas...queste cose qui ... ha fatto si che si pronuncino alla francese... ma in effetti bisognerebbe pronunciarli alla francoprovenzale.

I più hanno imperniato le loro argomentazioni sullo spostamento del baricentro del repertorio sociolinguistico locale, facendo riferimento agli effetti del contatto delle parlate locali con la lingua nazionale:

[P15]: [la pronuncia] è influenzata dall'italiano... quindi... potrebbe essere un fenomeno leggermente negativo perché... in fondo si va... a modificare un po' ehm... i nomi della Valle d'Aosta.

[P29]: credo che ci siano fenomeni di italianizzazione, tipo / kwart/ per / kar/o / Sarven'so per / Sarvan'so/.

[P 108]: Penso che l'italianizzazione in seguito al ventennio fascista dei nomi delle località valdostane può aver poi influito anche in questii ...mhh... nella... nella pronuncia, perché poi magari quando sono tornati al francese in realtà... ci sono anche delle... come dire? ... tra il francoprovenzale e il francese... delle interferenze, che quindi possono influire.

Processi di italianizzazione sono stati occasionalmente ricondotti ai provvedimenti autarchici del regime fascista, ben radicati nell'immaginario collettivo - specie dei più giovani - benché in realtà di impatto solo relativo sulle denominazioni dei Comuni (1)

[P27] questa varietà ha delle ragioni, perché penso che tipo a un certo punto avevan cambiato i nomi dei Comuni, no? anche durante il fascismo...che era ... quindi... non so se magari ad alcuni è rimasto in un modo, ad alcuni è rimasto in un altro

[P36] alcuni nomi vengono pronunciati un po' all'italiana... sono reminiscenze del fascismo...

Circa un terzo degli intervistati ha invece attribuito le pronunce disallineate alle condizioni di italofonia che caratterizzano tipicamente coloro che non appartengono alla comunità locale:

[P69]: la pronuncia dipende: se uno parla patois, se uno ... si è poi inserito qui in Valle d'Aosta dopo tende a italianizzare $i$ nomi. Oppure noi diamo invece un nome piuttosto patoisant ... dipende ... o francese, dipende, insomma.

[P 72]: c'è tanta gente che viene da fuori...: non mi disturba particolarmente sentirli pronunciare in altro modo.

[P 107]: eh, bisognerebbe capire perché ci sono tutte queste varianti... pensando a tutte le varianti di patois che ci sono... sarebbe interessante capire ... cioè, capire come mai ci sono tutte queste differenzeee... questi modi 
Toponimi e identità: la percezione dei parlanti valdostani.

L. REVELLI

DIATOPIA INTERNA E INTERTOLLERANZA DELLE VARIANTI diversi di pronunciare, se sono magari influenzati dall'italiano o meno, insomma...

[P115]: li penso che sia un pochettino...cioè... magari una questione anche di famiglia, magari eh... chi è radicato, eh... che parla patois, probabilmente lo dice cosi come si dice tradizionalmente e invece chi magari viene da fuori... anche solo di un generazione... ma i genitori lo dicevano in un certo modo... allora loro lo prendono di conseguenza.

[P128]: la varietà di pronuncia dipende dal fatto che la persona che pronuncia sia o meno patoisant: se non sai il dialetto lo pronunci in un determinato modo, se parli il dialetto lo pronunci con gli accenti del tuo dialetto.

In alcuni casi, sono stati gli stessi immigrati da altre regioni italiane ad attribuirsi una condizione di alterità coincidente con pronunce difformi dai canoni endogeni:

[P19] io comunque ad esempio anche se sono nato qua non parlo benissimo il francese ... comunque ho una famiglia meridionale...: il francese lo uso in modo scolastico.

[P129] [la varietà di pronuncia] è normale perché comunque rispetta quella che è la provenienza linguistica delle persone, per cui ... ad Aosta non siamo tutti valdostani, io non lo sono per prima, quindi...

La mancata condivisione di norme di pronuncia è stata interpretata da circa un terzo degli intervistati come risultato, naturale e positivo, della frammentazione dialettale che tradizionalmente caratterizza l'area:

[8]: secondo me va anche tanto magari daaaa... dal patois che si parla, per quello un po' l'accento e... le varie cose.

[P15]: [la pronuncia] è influenzata dai diversi patois, che ciascuno pronuncia un po' a modo suo.

[P70]: la diversità delle pronunce è data dalla diversità dei patois... e il francese... e quindi ci sono delle pronunce diverse secondo me per questo motivo.

[P 105]: i nomi dei paesi vengono pronunciati in modo diverso perché dipende dal patois: perché il patois è diverso da una zona all'altra.

[P126]: chi ha una parlata di patois dell'alta Valle o della bassa Valle tende a dare un'accentazione diversa ai nomi di Comune.

Alcuni intervistati si sono rivelati non soltanto ben consci della sussistenza di fenomeni di diatopia interna, ma anche consapevoli di alcune conseguenti implicazioni psicolinguistiche:

[P125]: che poi la cosa della varietà della pronuncia dei nomi dei Comuni vale per gli stessi valdostani, ad esempio a mia madre, che è di /mon'dziove/, lei che ha il suo patois, no? gli dà fastidio sentire un altro patois: gli da molto fastidio. Quando lei sente il patois, che ne so, di /'kJna/ - che poi mia nonna era di /'konə/ - e allora gli da molto fastidio. Lei probabil- 
Toponimi e identità: la percezione dei parlanti valdostani.

L. REVELLI

\section{CONCLUSIONI}

mente ha la pronuncia ... sicuramente diversa rispetto a alcuni altri paesi.In questo senso, la tolleranza delle varianti di pronuncia è apparsa correlata, specie nei parlanti dialettofoni, a una rappresentazione del patois come codice polinomico, ossia come lingua «dont l'unité est abstraite et résulte d'un mouvement dialectique et non de la simple ossification d'une norme unique, et dont l'existence est fondée sur la décision massive de ceux qui la parlent de lui donner un nom particulier et de la déclarer autonome des autres langues reconnues» (MARGELLESI 1983: 314): la presenza di fenomeni di differenziazione interna è stata in questi casi descritta come valore rappresentativo del particolarismo e delle tradizioni locali, da mantenere e preservare:

[P 87]: le varietà di pronuncia secondo me ... come tutte le peculiarità della Valle d'Aosta... son cose che danno autenticità e caratterizzazione al territorio: meno male che ancora ci sono... sono legate al discorso dei diversi patois.

[P 94]: La varietà è un fatto di tradizione, come posso dire.... eh, dipende dai patois che... che usano nelle diverse zone, che sono diversi. Ognuno ha le sue tradizioni.

I risultati dell'inchiesta condotta sembrano suggerire che dalle rappresentazioni dei parlanti a riguardo della polimorfia toponomastica trapelino atteggiamenti contrastanti nei confronti del plurilinguismo. In generale, i risultati del contatto fra differenti codici vengono accolti in modo molto favorevole se ritenuti endogeni e lontani nel tempo: quando ricondotta a fasi storiche remote l'eterogeneità è considerata come valore da preservare perché simbolico di una tradizionale frammentazione autoctona e del particolarismo locale. Gli esiti del plurilinguismo più recente sono percepiti con maggiore cautela o anche decisa diffidenza: emerge diffusamente la preoccupazione che si possa trattare di contaminazioni di forme cristallizzatesi in un'età considerata aurea o comunque assunta come punto di riferimento normativo per la definitiva validazione delle pronunce "corrette".

La sovrabbondanza di forme alternative viene comunque colta e abitualmente utilizzata dai parlanti come risorsa. Soltanto se sollecitati alla riflessione metalinguistica molti manifestano sentimenti di incertezza o imbarazzo: la convinzione che per ciascun toponimo possa esistere un'unica forma esatta di denominazione induce a ritenere che la polimorfia costituisca, malgrado il contesto plurilingue, una violazione da censurare.

Gran parte del campione intervistato tende a giudicare più for- 
Toponimi e identità: la percezione dei parlanti valdostani.

L. REVELLI mali, prestigiose o corrette le soluzioni maggiormente distanti dall'italiano. Soprattutto le giovani generazioni - uniformemente e sistematicamente esposte all'insegnamento scolastico del francese - interrogate a proposito dell'esattezza di singole varianti formali selezionano senza distinzioni quelle che meglio rispondono ai canoni fonetici del modello d'Oltralpe: sugli usi, sulle soluzioni grafiche e sugli esiti effettivi della trafila francoprovenzale prevalgono, in sostanza, le rappresentazioni di correttezza veicolate dalla scuola. In reazione al patois, d'altra parte, alcuni parlanti dialettofoni, specie se anziani, introducono dispositivi di allontanamento che di fatto contraddicono il sentimento linguistico emergente in termini di affezione al territorio e alle sue tradizioni.

Si osserva così, da un lato, l'utilizzo spontaneo e abituale di forme la cui correttezza viene poi disconosciuta a livello riflessivo; d'altro lato, all'interno delle produzioni maggiormente sorvegliate, l'attivazione di dispositivi in alcuni casi anche ipercorretti, che - attribuendo alle varianti formali differenti ranghi diafasici - tendono a condizionare la spontaneità delle produzioni in proporzione alla formalità del contesto situazionale e alle presunte aspettative degli interlocutori.

Gli elementi di contraddittorietà che emergono tra intenzioni e comportamenti, tra scelte esibite e motivazioni addotte, tra giudizi di grammaticalità e valutazioni di accettabilità forniscono un quadro multiforme, al cui interno non si evidenziano tuttavia segni fattuali di insofferenza, tabuizzazione, sanzione o conflittualità: l'impressione generale è anzi che - anche in presenza di asserti di principio perentoriamente ostili alla polimorfia - la tolleranza delle varianti sia in realtà molto elevata e la compresenza di soluzioni alternative, anche nell'ambito delle produzioni di un medesimo parlante, passi nella quotidianità di fatto inosservata.

Risulta di conseguenza molto difficile, allo stato attuale, ipotizzare che le spinte alla standardizzazione emotivamente o programmaticamente auspicate da alcuni possano portare a breve alla propagazione di esiti uniformi. Se in un futuro, vicino o lontano, sulle variegate realizzazioni orali individuali riusciranno a imporsi soluzioni omogeneamente condivise sarà di certo interessante verificare se le forme prevalse saranno quelle conformi alle (super)norme sostenute dagli interventi di regolamentazione delle grafie condotti a livello amministrativo, funzionali a connotare un'identità forgiata sui canoni di una tradizione locale tuttavia per i parlanti al contempo troppo ingombrante e troppo 
Toponimi e identità: la percezione dei parlanti valdostani.

L. REVELLI

\section{RIMANDI BIBLIOGRAFICI}

evanescente; se saranno stati selezionati, piuttosto, gli esiti sostenuti dalle rappresentazioni di correttezza e prestigio veicolate dal canone scolastico, conformi alla matrice francofona anche quando in contraddizione con la trafila francoprovenzale; se si saranno magari imposte, invece, scelte alternative, legittimate dalla frequenza d'uso, semplificate o meticcie e proprio per questa ragione percepite come non marcate, e spontaneamente validate dalla comunità perché più comunemente scelte, praticate e condivise all'interno dell'eterogeneo repertorio dei parlanti.

ANTONINI F. \& MORETTI B. 2000. Le immagini dell'italiano regionale, Locarno, Dadò.

BERRUTO G. 2003. Una Valle d'Aosta, tante Valli d'Aosta? Considerazioni sulle dimensioni del plurilinguismo in una comunità regionale, Fondation Emile Chanoux, Una Valle d'Aosta bilingue in un'Europa plurilingue, Aosta, Tipografia Valdostana, pp. 44-53.

DUBERTI N. \& REGIS R. 2011. Standardizzazione toponomastica in aree di confine: il caso di Roccaforte Mondovi, Nomi, luoghi, identità. Toponomastica e politiche linguistiche, Atti del Convegno Internazionale (Cividale del Friuli, 17-19 novembre 2011), Udine, Società Filologica Friulana, in stampa.

MARCELLESI J.-B. 1984. La définition des langues en domaine roman: les enseignements à tirer de la situation corse, pp. 307314 in Bouvier J.-C. (a cura di), Sociolinguistique des langues romanes, Actes du XVIIème Congrès International de Linguistique et de Philologie Romanes (Aix-en-Provence, 29 août-3 septembre 1983), vol. 5, Aix-en-Provence/Marseille, Université de Provence-Jeanne Laffitte.

MATURI P. 2006. I suoni delle lingue, $i$ suoni dell'italiano, Bologna, Il Mulino.

PUOLATO D. 2006. Francese-italiano, italiano-patois: il bilinguismo in Valle d'Aosta fra realtà e ideologia, Bern, Peter Lang.

RAIMONDI G. 2003. La toponomastica. Elementi di metodo, Torino, Libreria Stampatori. 
Toponimi e identità: la percezione dei parlanti valdostani.

L. ReVELLI
RAIMONDI G. 2006. Storia e configurazione del repertorio plurilingue valdostano, pp. 100-126 in BERTOLINO F., REVELLI L. (a cura di), Università, Scuola, territorio, Milano, Franco Angeli.

REVELLI L. 2013. Polimorfia e (ri-)standardizzazione della toponomastica in contesto alloglotto: il caso della Valle d'Aosta. in GALVO RIGUAL C., MINERVINI L., THIBAULT A. (a cura di), Actes du XXVIIe Congrès international de linguistique et de philologie romanes (Nancy, 15-20 juillet 2013). Section 11 - Linguistique de contact - publication électronique, Nancy, ATILF, in stampa.

TELMON T. 2002. Le ragioni di un titolo, pp. V-XXXIV in CINI M., REGIS R. (a cura di) Che cosa ne pensa oggi Chiaffredo Roux? Percorsi della dialettologia percezionale all'alba del nuovo millennio, Atti del Convegno Internazionale di studi (Bardonecchia, 25-27 maggio 2000), Alessandria, Edizioni dell'Orso.

\section{NOTA}

Il provvedimento di italianizzazione della toponomastica comunale, introdotto soltanto nel 1939 (R.D. 1442 "Riduzione in forma italiana delle denominazioni di trentadue Comuni della provincia di Aosta"), risulta nel periodo successivo disatteso non soltanto negli usi, ma spesso anche nei documenti ufficiali. 